\title{
SEMIGROUPS IN COMPACT GROUPS ${ }^{1}$
}

\author{
FRED B. WRIGHT
}

In the theory of one-parameter semigroups, the parameter family is usually an open semigroup of the real or complex numbers (under addition). Thus it is fundamental to investigate the structure of such semigroups. For a large class of these the structure is well known. These are the angular semigroups [3, Definition 7.6.1]; that is, those open semigroups which have the identity element 0 as a limit point. A complete discussion of angular semigroups of the real line and Euclidean 2-space will be found in [3, Chapter VII] and [4]. Furthermore, these methods of Hille and Zorn can be extended to a characterization of angular semigroups in Euclidean $n$-space $E^{n}$ [12].

In this paper the structure of all open semigroups in any compact topological group is determined. We can then extend this result to a classification of the angular semigroups of any abelian topological group $H$ which contains a compact open subgroup $K$. Coupling this with the results for Euclidean $n$-space, we obtain an essentially complete description of the angular semigroups of an arbitrary locally compact group.

For compact groups, the result is remarkably simple.

THEOREM I. Let $K$ be any compact group, and let $S$ be a semigroup in $K$. Then $S$ is necessarily a closed subgroup of $K$ under either of the following conditions: (1) $S$ is closed, (2) $S$ is open.

PROof. The case where $S$ is closed is already well known, and is contained in a theorem on topological semigroups due to several authors $[2 ; 5 ; 6 ; 8 ; 9 ; 13]$. The facts are these: $S$ is a topological semigroup, or mob, and is compact. Then $S$ must contain idempotents, and $S$ is a topological group if and only if there is an identity element and there are no other idempotents in $S$. It is clear that these conditions are satisfied in the present case, and hence $S$ is a group.

The case where $S$ is open reduces to this. For, let $H=\bar{S}$; then $H$ is a closed semigroup, and therefore a group. If the identity element 1 of $K$ is in $S$ and isolated in $S$, then it is isolated in $K$, and therefore $K$ is discrete and finite. Either the standard algebraic result [15, Theorem 1, p. 3 ] or the above remarks then imply that $S$ is a group. Otherwise, 1 is a limit point of $S$, and $S$ is therefore angular. By [3, Theorem 7.6.2 ], we have $S=H^{0}$, where $H^{0}$ denotes the interior of $H$. But

Received by the editors April 25, 1955.

${ }^{1}$ Written under contract N7-onr-434, Task Order III, Office of Naval Research. 
any subgroup of a topological group having a nonvoid interior is an open and closed subgroup, so that $S=H^{0}=H$, q.e.d.

We may now appeal to the Zorn Category Theorem [3, Theorem 7.7.1] for the following consequence, which bears a striking resemblance to well known theorems of Banach [1, Theorem 1, p. 21] and Kuratowski [3, Theorem 1.8.1].

THEOREM II. Let $K$ be a compact group and let $S$ be a semigroup in $K$ which is of the second category at the identity 1 of $K$ and which satisfies the condition of Baire. Then $S$ is a subgroup of $K$ which is both open and closed.

Proof. Zorn's theorem states that the interior $S^{0}$ of $S$ is dense in $S$ and that $S^{0}$ is the interior of $\bar{S}$. But $S^{0}$ is clearly a semigroup, and is not empty since $S$ is not. Then Theorem I applies: $S^{0}=S=\bar{S}$.

Slightly more general versions of the category theorem will be found in $[7 ; 10]$.

A further result, reminiscent of the situation in locally compact groups, is the following immediate consequence of Theorem I.

THEOREM III. In a compact group $K$, any semigroup $S$ which is locally compact in its induced topology is a subgroup of $K$ which is both open and closed.

Now let $H$ be an abelian topological group containing a compact open subgroup $K$, and let $S$ be any open semigroup in $H$. If $S \cap K$ is not empty, then $S \cap K$ is an open subgroup of $K$, and hence of $H$, by Theorem I. On the other hand, if $S \cap K$ is empty, then $S$ is not angular, since $K$ is a neighborhood of the identity 1 of $H$. If $S \neq S \cap K$, let $x \in S, x \notin K$. Since $S$ is a semigroup we have $x(S \cap K) \subset S$. This shows that if $S^{*}$ is the image of $S$ in $H / S \cap K$, then the complete inverse image of $S^{*}$ is simply $S$ again. Clearly $S^{*}$ contains the identity of $H / S \cap K$. Conversely, if $K_{1}$ is any open subgroup of $K$, and if $S^{*}$ is any semigroup in the (discrete) group $H / K_{1}$ which contains the identity, then the complete inverse image of $S^{*}$ in $H$ is an angular semigroup of $H$.

If $G$ is any locally compact abelian group, then there exists a direct product decomposition $G=E^{n} \times H$ of $G$, where $E^{n}$ is a Euclidean space of unique dimension $n$ and where $H$ is a group containing a compact open subgroup $K[11 ; 14]$. Then an angular semigroup of $G$ is the direct product of angular semigroups in $E^{n}$ and $H$. The above discussion, coupled with the results for Euclidean spaces, thus gives an essentially complete description of the angular semigroups of $G$. 


\section{REFERENCES}

1. S. Banach, Theorie des operations lineaires, Warsaw, 1932.

2. B. Gelbaum, G. K. Kalish, and J. M. H. Olmstead, On the embedding of topological semigroups and integral domains, Proc. Amer. Math. Soc. vol. 2 (1951) pp. 807-821.

3. E. Hille, Functional analysis and semi-groups, Amer. Math. Soc. Colloquium Publications vol. 31, New York, 1948.

4. E. Hille and M. Zorn, Open additive semigroups of complex numbers, Ann. of Math. (2) vol. 44 (1943) pp. 554-561.

5. K. Iwasawa, Finite and compact groups, Sugaku vol. 1 (1948) pp. 30-31 (Japanese).

6. R. J. Koch and A. D. Wallace, Maximal ideals in compact semigroups, Duke Math. J. vol. 21 (1954) pp. 681-686.

7. E. J. McShane, Images of sets satisfying the condition of Baire, Ann. of Math. (2) vol. 51 (1950) pp. 380-386.

8. K. Numakura, On bicompact semigroups, Math. J. Okayama Univ. vol. 1 (1952) pp. 98-100.

9. J. E. L. Peck, Yale University Dissertation, 1950.

10. B. J. Pettis, Remarks on a theorem of E. J. McShane, Proc. Amer. Math. Soc. vol. 2 (1951) pp. 166-171.

11. L. Pontrjagin, Topological groups, Princeton, 1946.

12. R. A. Rosenbaum, Subadditive functions, Duke Math. J. vol. 17 (1950) pp. 227-247.

13. A. D. Wallace, $A$ note on mobs. II, Anais da Academia Brasileira de Ciencias vol. 25 (1953) pp. 335-336. 1940.

14. A. Weil, L'integration dans les groupes topologiques et ses applications, Paris,

15. H. Zassenhaus, The theory of groups, New York, 1949.

TUlane University of Louisiana 